\title{
Blind Separation of Abdominal Electrocardiogram Sources through Dynamic Neural Network
}

\author{
Dmitriy Devyatykh \\ Applied Mathematics department \\ National Research Tomsk Polytechnic University \\ Tomsk, Russia \\ ddv.edu@gmail.com
}

\begin{abstract}
Cardiovascular system of the fetus is biological critical infrastructure. Fetal electrocardiogram and its characteristics such as heart ratio, waveform and dynamic behavior overall include vital information about health state, development and possible deviations from normal fetation. Thus fetal heart ratio monitoring is mandatory during pregnancy. Widespread Doppler ultrasound diagnostics can guarantee obtaining accurate results but is not suitable for long-term monitoring. Non-invasive fetal electrocardiography proposes to extract fetal signal from maternal abdominal electrocardiogram. This approach is applicable for long-term monitoring, but because of amplitude of maternal R-peaks is significantly larger than fetal it is a challenge to extract fetal signal. In this paper we propose using dynamic neural networks for extracting fetal components and demonstrate its advantages compared to blind source separation though independent component analysis. The training algorithm is a combination of backpropagation through time and resilient propagation. The proposed approach accuracy of R-peak detection is $97 \%$. Statistical analysis proved that developed algorithm can process even non-stationary signals with loss of accuracy and no additional training is required.
\end{abstract}

Keywords-dynamic neural network; vanishing gradient; blind source separation; fetal electrocardiogram; resilient propagation

\section{INTRODUCTION}

Fetal heart activity monitoring is vital procedure as it allows not only to estimate current health state of the fetus but to prevent deviation from normal fetation process.

Non-invasive extraction of fetal electrocardiogram (FECG) from abdominal electrocardiogram (AECG) of mother is challenging task. The signal is obscured with various interferences and maternal electrocardiogram (MECG) that has R-peaks with significantly larger amplitude. Despite the difficulties, developing of such approach is required by modern medicine as it provides greater possibilities for long-term monitoring of fetal heart activity. Although Doppler ultrasound diagnostics procedure can guarantee obtaining accurate results such drawbacks as negative effect of the ultrasound on a fetus and intermittent reposition of the sensor reduce capabilities of long-term monitoring[1]. According to history of non-invasive fetal electrocardiography development and current state of art we can distinguish several general groups of methods.

\author{
Olga Gerget \\ Applied Mathematics department \\ National Research Tomsk Polytechnic University \\ Tomsk, Russia \\ gerget@gmail.com
}

Obvious way to extract FECG would be to find its unique characteristics in spectral domain. Unfortunately, maternal and fetal components often overlap in both spectral and time domains [2]. Moreover, in many cases overlapped R-peak of mother and fetus does not possess such amplitude that would prevail over close maternal R-peaks. In [3] authors proposed special triple parametric transform suitable for estimating fetal heart ratio (FHR). The key feature of proposed transform was using square-wave analyzing functions instead of harmonics This method did not solve the task of separating sources, as maternal R-peaks were preliminary deleted in manual way, so the proposed transform could be applicable for FECG but not AECG processing. Although the method was capable of correct FHR determining even when fetal R-peaks were not visible, the accuracy of such procedure drops significantly with RR-variability growth.

Because of non-stationary nature of electrocardiograms (ECG) it is hard pro process them using Fourier based approaches. Classic solution lies in using Wavelet transform. Several papers [4-5] demonstrate suitability of Waveletbased approach for filtering high and low end frequencies, but the separation itself is usually performed by pattern recognition methods such as pattern matching or neural network. Extracting FECG though wavelet-zoom procedure and Lipschitz exponents calculating possesses severe drawback - this method does not allow to separate maternal and fetal R-peaks if they are overlapped in time domain [6]. Depending on individual ECG characteristics of a person it increases mistake by $10 \%-20 \%$.

Non-invasive fetal electrocardiography was pioneered by Widrow [7] who proposed using linear adaptive filters for solving the task. The method proposes finding FECG through deleting MECG from thoracic channel, that is considered as reference signal, from AECG, that is considered as main input signal. Such approach demands high similarity between maternal QRS shapes in reference and input signals [8-9]. Wrong electrode placement or even small containment may bring distortion into morphological properties of R-peak, thus reducing convenience of method. Such method is not suitable for blind source separation as it requires prior knowledge about maternal source of AECG. Nowadays most datasets [10] include multichannel AECG but adaptive filtering may use only one of them as main signal. 
Involving of artificial neural network into adaptive linear filtering allowed to implement linear and non-linear mappings between MECG and AECG. Most of papers [1113] propose using models based on multi-layer perceptron (MLP). This model is static and does take into account timevarying features of maternal and fetal mixing process.

Blind source separation (BSS) proposes unique approach for extracting sources without prior knowledge about signals or mixing process. It supposes linear way of mixing source signals using mixing matrix. The separation may be performed with separating matrix that is inverse to mixing matrix. Because both matrices are unknown finding of dividing matrix is performed though independent component analysis (ICA) that involves maximizing negentropy approach. ICA possesses several drawbacks. The accuracy depends on amount of independent mixes and their length. The method supposes that mixing is performed in linear and static way which is not always correct for AECG [14]. Each time the algorithm is performed rows of matrix of sources are randomly located related to each other that complicates algorithmization of windowed processing.

\section{MATERIALS AND METHODS}

The cornerstone idea of our approach is to use weight matrices of dynamic neural network as dividing matrices in blind source separation. Features of neural network architecture allow to find origin of the source, because each output neuron may be train to extract exact source from mix. Extraction of FECG may be performed using even single channeled input AECG.

In order to be dynamic neural network has to possess some kind of short-term memory. It may take form of input delays or feedbacks. Thus, previous input and output values may be used for current output calculation. Analysis of several independent papers [15-17] showed that amongst various architectures of networks such as focused time delay, distributed time delays, Jordan, Elman, Hopfield, Hamming, the most suitable architecture for time-serious processing is non-linear autoregressive model with exogenous inputs (NARX). Basically it is multilayer perceptron with shortterm memory. Input neurons of such network are supplied with input delays, output neurons are connected with hidden layer neurons with feedbacks.

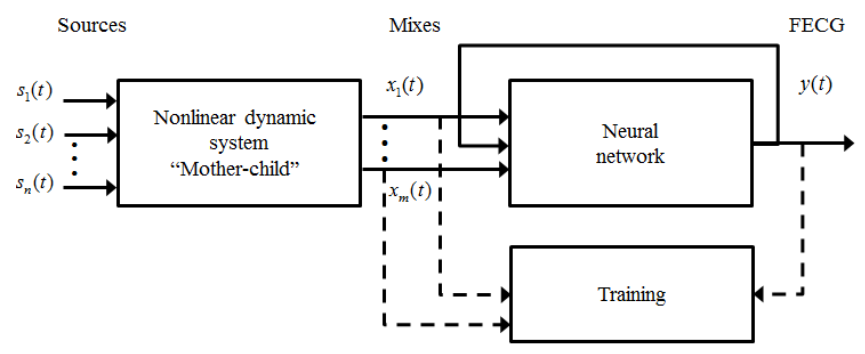

Fig. 1. Blind source separation based on non-linear autoregressive model with exegenous inputs

Equation (1) describes overall dynamics of the NARX

$y(t)=f(u(t), u(t-1), u(t-2), \ldots, u(t-p), y(t-1), y(t-2), \ldots, y(t-q))$, where output value $y$ at $t$ moment of time is calculated as function $f$ of $p$ previous inputs $u$ and $q$ previous outputs.

First step that has to be taken before training of dynamic network starts is unfolding. It allows save dynamics of recurrent network, but all feedbacks are replaced with straight-forward connections. Depending on amount of values in time-series, structure of network is cloned and added to existing one. As a result dynamic neural network is transformed into multi-layer perceptron with significant amount of hidden layers. Standard backpropagation is not suitable for calculating gradients of unfolded network. Because some of hidden unfolded neurons originated from output neurons they can be considered as output and hidden the same time. So when calculating gradient for such neuron it has to be considered that desired output for such neuron is known and we can calculate error, but because there next layers principle of backpropagation should be applied too. Thus calculating of local gradient for hidden neuron of unfolded neuron may be described with (2)

$$
\delta_{i}^{k}(t)=\left\{\begin{array}{l}
\varphi^{\prime}\left(v_{i}^{k}(t) e_{i}^{k}(t)\right), \quad k=L \\
\varphi^{\prime}\left(v_{i}^{k}(t)\right)\left[e_{i}^{k}(t)+\sum_{j \in A} w_{i j}^{k} \delta_{i}^{k+1}(t+1)\right], \quad k \in O \\
\varphi^{\prime}\left(v_{i}^{k}(t)\right)\left[\sum_{j \in A} w_{i j}^{k} \delta_{i}^{k+1}(t+1)\right], \quad k \in H
\end{array}\right.
$$

where:

- $\quad w$ weight that connects $i$-th neuron from current $k$ layer with $j$-th neuron from next layer;

- $\varphi, \varphi^{\prime}$ activation function of neuron and its derivative;

- $v$ linear combination of input and weight vectors

- $e$ subtraction real output of neuron from target value

- $O, H$ set of unfolded hidden layer originated from output and hidden neurons respectively

- $\delta$ local gradient of neuron, which is derivative of minimizing function with respect to $v$.

- $L$ length of input signal from training set, if $k=L$ then current layer is considered as output.

- $A$ set of neurons from $k+1$ layer connected with current neuron

After gradients of neurons are calculated the weight corrections may be found according to any gradient-based training algorithm.

Training of unfolded dynamic networks faces the same problem that deep neural network do[18]. It comes from linearity of backpropagation and is called vanishing/exploding gradients problem. Maximum of the derivative of sigmoid activation function illustrated at fig. 2 is 0.25. Standard initialization of weight uses normal 
distribution, thus $\left|w_{i j}^{k}\right|<1$. Obviously multiplication of activation function derivative and weight will be less than 0.25 .

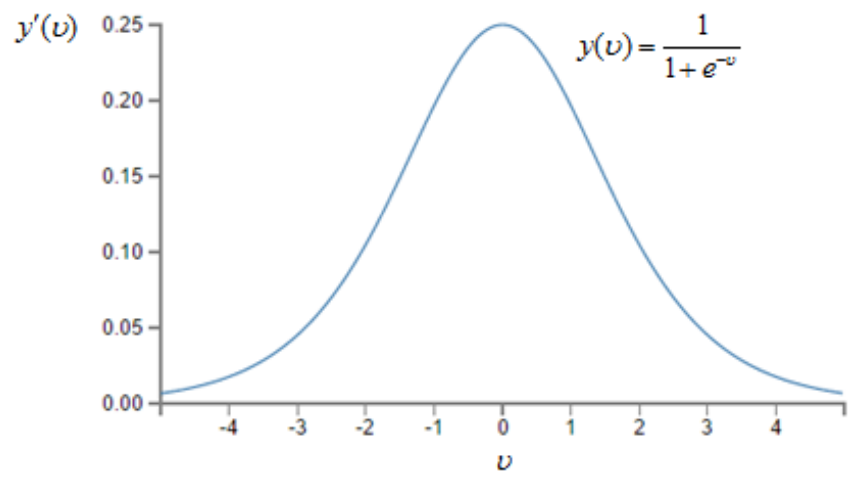

Fig. 2. Derivative of sigmoid activation function

Thus backpropagation causes permanent vanishing of local gradients that may be described with (3)

$$
\delta_{i}^{k}=\varphi^{\prime}\left(v_{i}^{k}\right) \sum_{j \in A} \overbrace{w_{i j}^{k} \delta_{j}^{k+1}}^{<0.25|A|} .
$$

Consequence of gradient decay lies in tiny correction of weights that are located far from output layer [19]. Dynamics of weight behavior during training process is illustrated at fig. 3, we can see that maximum change of first hidden layer weights is the same order as minimum change of fourth hidden layer neurons. Thus only part of weights can be used for adapting of neural network. Moreover neurons near output layer will have to change their weight according to data that was already distorted by untrainable neurons near input layer.

In order to solve problem of long-term dependency training of unfolded dynamic networks we used resilient propagation algorithm to calculate weight correction. It does not solve fundamental problem of backpropagation, but because value of gradient is not used for calculating of new weight, such approach is suitable.

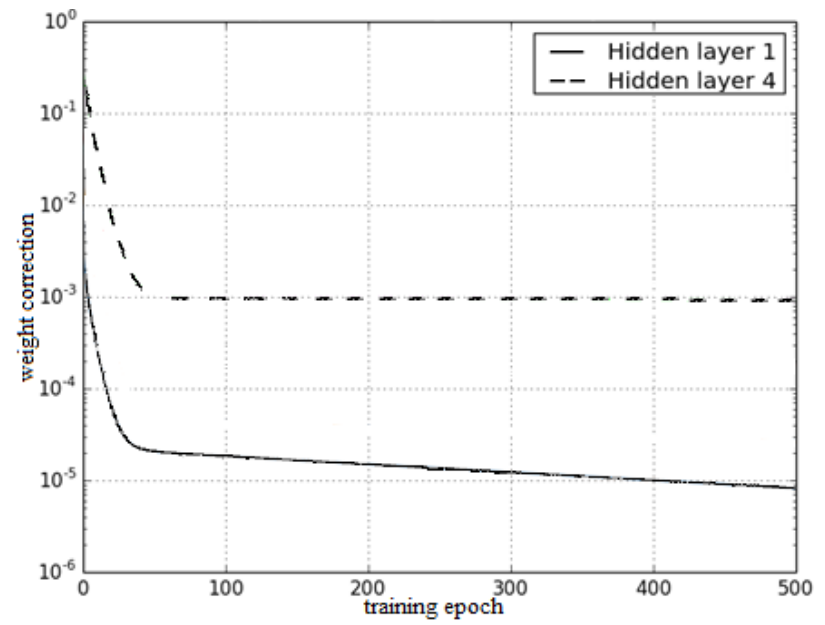

Fig. 3. Correction of weight according to layer of hidden neuron
Resilient propagation [20] uses sign of derivative instead of its value is calculated using (4)

$$
\Delta w_{i j}(t)=\left\{\begin{array}{l}
-\Delta_{i j}^{(t)} \eta, \frac{\partial E^{(t)}}{\partial w_{i j}}>0 \\
+\Delta_{i j}^{(t)} \eta, \frac{\partial E^{(t)}}{\partial w_{i j}}<0 \\
0, \frac{\partial E^{(t)}}{\partial w_{i j}}=0
\end{array}\right.
$$

where $E$ is minimized function and $\eta$ is found using (5)

$$
\eta=\left\{\begin{array}{c}
\eta^{+}, \sum_{n=0}^{L} \frac{\partial E(n)^{(\mathrm{t})}}{\partial w_{i j}} \cdot \sum_{n=0}^{L} \frac{\partial E(n)^{(\mathrm{t}-1)}}{\partial w_{i j}}>0 \\
\eta^{-}, \sum_{n=0}^{L} \frac{\partial E(n)^{(\mathrm{t})}}{\partial w_{i j}} \cdot \sum_{n=0}^{L} \frac{\partial E(n)^{(\mathrm{t}-1)}}{\partial w_{i j}}<0 \\
0<\eta^{-}<1<\eta^{+},
\end{array},\right.
$$

where $k$ denotes index of current value from time-series input data and $t$ denotes epoch of training process.

The dataset we used for this paper was acquired from physionet.org database. Representativeness of ECGs was proved by medical experts from Siberian State Medical University and Cardiology Research Institute. The dataset included 5-channeled ECG signals of 25 pregnant women. First four channels were AECGs, the fifth was FECG obtained through invasive procedure. Sampling rate was 1 $\mathrm{kHz}$, length of each person record was 1 minute long. Total amount of R-peaks was 4930, each of them was characterized as fetal, maternal or overlapped. The whole dataset was divided into training and testing sets. 10 seconds long pieces from 12 out of 25 people were used for training, the rest data was used for testing. Half of the people from dataset did not take part in training at all, thus we prevented networks from overfitting. Validation set was not used, because it uses mean square error as mark of quality of training, while we used amount of R-peaks recognized by network as measurement of accuracy. Example of input signal and corresponding target signal is demonstrated in fig. 4.
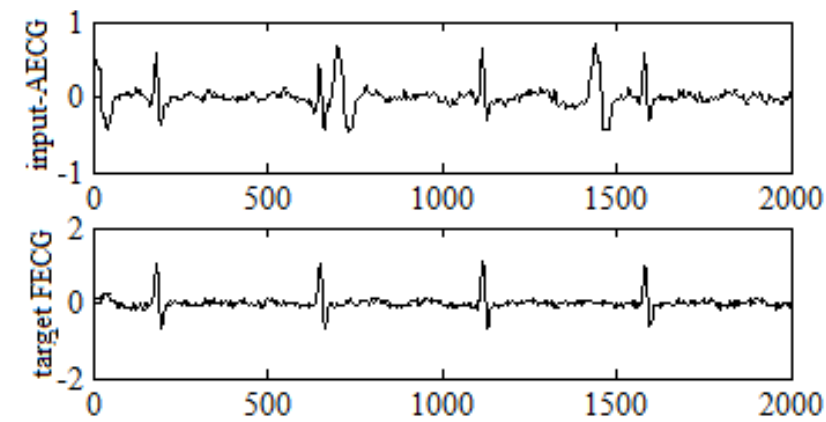

Fig. 4. Input abdominal signal and desired output fetal signal 
Training-testing sets existed in two versions: singlechanneled AECG; four-channeled; thus we experimented with dimension of input signal and its influence on accuracy of network.

\section{RESULTS}

Parameters that we used to adjust network were amount of: hidden neurons; input delays, denoted as $p$; feedbacks, denoted as $p$. Activation function of hidden neurons was hyperbolic tangent, because output of this function belongs to $(-1 ; 1)$.

At first we designed neural networks with minimum amount of input delays and feedbacks, and then we gradually increased value of parameters of network until accuracy of FECG extraction stopped growing or started decaying.

Single-channeled AECGs were processed with best accuracy by neural network with 10 input delays and 10 feedbacks. Table 1 includes results of recognizing R-peaks. When value of these parameters was lower than 5 , only 10 out of 25 FECGs were extracted with relatively high accuracy. Successfully processed signals had the highest signal-noise ratio (SNR), which was 0.7-0.6, while others AECG had 0.15-0.12 SNR. Absolute amount of mistakes was evenly distributed between all three groups of R-peaks, but relatively overlapped R-peaks with worst accuracy. Networks with single hidden neuron produced output signal with same as input, thus we considered such result as $0 \%$ accurate.

Four-channeled AECGs were processed with best accuracy by neural network with 5 input delays and 5 feedbacks. Table 2 includes results of recognizing R-peaks. Accuracy of extracting FECG from multichannel data by network even with 1 input delay and 1 feedback was higher than accuracy of any network, trained on single-channel signal. Network with minimum of short-term memory succeeded in extracting FECG from signals with 0.6-0.7 SNR even with one hidden neuron. Increasing of accuracy was mostly by correct recognizing of overlapped R-peaks.

To answer the question whether R-peaks from output of neural network are placed in same place in time as in desired output we used statistics. We build RR-intervals for ECG from output and target signals. Null hypothesis that RRintervals come from a population with mean equal to zero was proven by paired t-test.

TABLE I. EXTRACTING FECG FROM SINGLE-CHANNELED AECG WITH 10 INPUT DELAYS AND 10 FEEDBACKS

\begin{tabular}{|c|c|c|c|c|}
\hline \multirow{2}{*}{$\begin{array}{c}\text { Hidden } \\
\text { neurons }\end{array}$} & \multicolumn{4}{|c|}{ R-peaks detected } \\
\cline { 2 - 5 } & $\begin{array}{c}\text { fetal } \\
(\mathbf{2 8 2 0})\end{array}$ & $\begin{array}{c}\text { maternal } \\
(\mathbf{1 7 3 5 )}\end{array}$ & $\begin{array}{c}\text { overlapped } \\
(\mathbf{3 7 5})\end{array}$ & $\begin{array}{c}\text { total } \\
(\mathbf{4 9 3 0})\end{array}$ \\
\hline 1 & 0 & 0 & 0 & $0 \%$ \\
\hline 5 & 2567 & 1510 & 175 & $86 \%$ \\
\hline 10 & 2581 & 1505 & 180 & $86 \%$ \\
\hline 15 & 2632 & 1465 & 200 & $87 \%$ \\
\hline
\end{tabular}

We created several artificial AECGs with significant RRvariability. These signals were based on real AECGs from testing data set, but distance between R-peaks was changed.
TABLE II. EXTRACTING FECG FROM SINGLE-CHANNELED AECG WITH 2 INPUT DELAYS AND 2 FEEDBACKS

\begin{tabular}{|c|c|c|c|c|}
\hline \multirow{2}{*}{$\begin{array}{c}\text { Hidden } \\
\text { neurons }\end{array}$} & \multicolumn{4}{|c|}{ R-peaks detected } \\
\cline { 2 - 5 } & $\begin{array}{c}\text { fetal } \\
(\mathbf{2 8 2 0})\end{array}$ & $\begin{array}{c}\text { maternal } \\
(\mathbf{1 7 3 5 )}\end{array}$ & $\begin{array}{c}\text { overlapped } \\
\mathbf{( 3 7 5 )}\end{array}$ & $\begin{array}{c}\text { total } \\
\mathbf{( 4 9 3 0 )}\end{array}$ \\
\hline 1 & 1160 & 685 & 130 & $40 \%$ \\
\hline 5 & 2720 & 1625 & 330 & $95 \%$ \\
\hline 10 & 2739 & 1604 & 334 & $95 \%$ \\
\hline 15 & 2757 & 1659 & 340 & $97 \%$ \\
\hline
\end{tabular}

Mann-Whitney $U$-test was used to prove null hypothesis that RR-intervals of network's output and artificial AECG with RR-variability come from same population. Moreover accuracy of extracting FECG from real signal and signal with artificial RR-variability was the same.

We successfully used trained neural networks for blind separating fetal source from AECG. To prove advantage of such approach we performed ICA extraction from same dataset. Linear approach extracted FECG with $100 \%$ accuracy only from 10 out of 25 records. These were AECG with highest SNR that 0.6-0.7. Separating sources from signals with such SNR was successful only in limited set of conditions:

- independent amount of observed mixes must not be lower than 3 ;

- length of signal should be less than 10 seconds.

ICA is not able to separate sources of AECG using less than 4-channel input signal. Separating of sources may be performed only in window-mode, while neural network may the whole AECG signal.

\section{CONCLUSIONS}

Blind source separation has more potential for extracting sources from mixed signals, as it requires no prior information and is more robust to personal characteristics of signal. Artificial neural network extracts fetal ECG more accurately compared to independent component analysis, as it is nonlinear and dynamic. The less "visible" fetal R-peak is, the more input delays and feedbacks it is required for successful extraction. Multichannel records are more convenient for FECG extraction. Neural network approach allows process non-stationary ECG signals without them being included into training dataset.

\section{REFERENCES}

[1] Noguchi, Y., Mamune, H., Sugimoto, S., Yoshida, J., et al. (1994) Measurement characteristics of the ultrasound heart rate monitor. Engineering in medicine and biology society. Engineering advances. New opportunities for biomedical engineers. Proceedings of the 16th annual international conference of the IEEE. Baltimore, MD, USA. 1, 670-671.

[2] Sameni R, Clifford GD. A Review of Fetal ECG Signal Processing; Issues and Promising Directions. Open Pacing Electrophysiol Ther J. 2010;3:4-20.

[3] Tal, Y., Akselrod, S. (1989) Fetal heart rate detection by a special transformation method. Proceedings of Computers in Cardiology 275-278, Jerusalem, Israel.

[4] Mochimaru F., Fujimoto Y. (2002) Detecting the fetal electrocardiogram by wavelet theory-based methods. Prog Biomed Res 7:185-193 
[5] Echeverria, J. C., Ramirez, N., Pimentel, A. B., et al. (1996) Fetal QRS extraction based on wavelet analysis and pattern matching. Engineering in medicine and biology society. Bridging disciplines for biomedicine. Proceedings of the 18th Annual International Conference of the IEEE. Amsterdam. Netherlands; 31 October-3 November 1996. vol. 4. p. 1656-1657.

[6] Hasan MA, Reaz MB, Ibrahimy MI, Hussain MS, Uddin J. Detection and Processing Techniques of FECG Signal for Fetal Monitoring. Biol Proced Online. 2009;11:263-95.

[7] Widrow B., Glover J. R. Jr, McCool J. M. et al. (1975) Adaptive noise canceling: principles and applications. Proc IEEE 63: 16921716.

[8] Kam, A., Cohen, A. (1999) Detection of fetal ECG with IIR adaptive filtering and genetic algorithms. IEEE International Conference on Acoustics, Speech, and Signal Processing (ICASSP'99) 4, 23352338. Phoenix, AZ, USA

[9] Stoughton J. W., Weber G. N., Pretlow R. A. (1990) Fetal heart rate estimation via adaptive least mean square linear prediction methods. Proc IEEE Southeastcon '90 1:260-264 New Orleans, LA, USA.

[10] Fuchs T, Pomorski M, Grobelak K, Tomiałowicz M, Zimmer M. Signal loss during fetal heart rate monitoring using maternal abdominal surface electrodes between 28 and 42 weeks of pregnancy. Adv Clin Exp Med. 2014;23(5):813-9.

[11] Jia, W., Yang, C., Zhong, G., Zhou, M. and Wu, S. (2010). Fetal ECG extraction based on adaptive linear neural network. 2010 3rd International Conference on Biomedical Engineering and Informatics.

[12] Ye, Y., Yao, X., Zhang, Z. and Mo, Q. (2007). A Non-Invasive Fetal Electrocardiogram Extraction Algorithm Based on ICA Neural Network. 2007 1st International Conference on Bioinformatics and Biomedical Engineering.

[13] Ma, Y., Xiao, Y., Wei, G. and Sun, J. (2014). Fetal ECG extraction using adaptive functional link artificial neural network. Signal and Information Processing Association Annual Summit and Conference (APSIPA), 2014 Asia-Pacific.

[14] M. Kotas, J. Jezewski, "Spatio-temporal Filtering for Fetal QRS Enhancement", World Congress on Medical Physics and Biomedical Engineering, September 7 - 12, 2009, Munich, Germany Volume 25/4 of the series IFMBE Proceedings pp 389-392

[15] Mandic, D. \& Chambers, J. (2001). Recurrent Neural Networks for Prediction: Learning Algorithms, Architectures and Stability. Wiley. ISBN 0-471-49517-4.

[16] Brezak, D., Bacek, T., Majetic, D., Kasac, J. and Novakovic, B. (2012). A comparison of feed-forward and recurrent neural networks in time series forecasting. 2012 IEEE Conference on Computational Intelligence for Financial Engineering \& Economics (CIFEr).

[17] Saroha, S. and Aggarwal, S. (2014). Multi step ahead forecasting of wind power by different class of neural networks. 2014 Recent Advances in Engineering and Computational Sciences (RAECS).

[18] Heaton, J. (2013). Deep learning and neural networks. St. Louis, MO: Heaton Research, Inc.

[19] Nielsen M. (2015). Neural Network and Deep Learning. $\begin{array}{lll}\text { Determination } & \text { press. } & \text { Retrieved }\end{array}$ http://neuralnetworksanddeeplearning.com/

[20] M. Riedmiller and H. Braun. A direct adaptive method for faster backpropagation learning: The RPROP algorithm. In H. Ruspini, editor, Proceedings of the IEEE International Conference on Neural Networks (ICNN), pages 586 \{ 591, San Francisco, 1993. 
